\title{
Light Exiting from Real Photonic Band Gap Crystals is Diffuse and Strongly Directional
}

\author{
A. Femius Koenderink* and Willem L. Vos \\ Complex Photonic Systems, Department of Applied Physics and MESA ${ }^{+}$Research Institute, University of Twente, \\ P.O. Box 217, 7500 AE Enschede, The Netherlands
}

(Received 31 October 2002; published 19 November 2003)

\begin{abstract}
Any photonic crystal is in practice periodic with some inevitable fabricational imperfections. We have measured angle-resolved transmission of photons that are multiply scattered by this disorder in strongly photonic crystals. Peculiar non-Lambertian distributions occur as a function of frequency: due to internal diffraction, wide angular ranges of strongly reduced diffuse transmission coincide with photonic stop bands, while enhancements occur for directions outside stop gaps. We quantitatively explain the experiment with a model incorporating diffusion and band structure on equal footing. We predict that in the event of a photonic band gap, diffuse light at frequencies near band gap edges can exit only along isolated directions. Angle-resolved diffuse transmission appears to be the photonic equivalent of angle-resolved photoelectron spectroscopy.
\end{abstract}

DOI: $10.1103 /$ PhysRevLett.91.213902

Currently many efforts are devoted to a novel class of dielectric composites known as photonic crystals [1]. Spatially periodic variations of the refractive index commensurate with optical wavelengths cause the photon dispersion relation to organize in bands, analogous to electron bands in solids. Generally, frequency windows known as stop gaps appear in which modes are forbidden for specific propagation directions. Fundamental interest in photonic crystals is spurred by the possibility of a photonic band gap, a frequency range for which no modes exist at all. Tailoring of the electromagnetic mode density by a photonic crystal allows one to control fundamental atom-radiation interactions in solid state environments $[2,3]$. In this context the hallmark of a photonic band gap is the eagerly awaited full inhibition of spontaneous emission [2]. Additional interest is aroused by the possibility of Anderson localization of light by point defects added to photonic band gap crystals [4].

Despite advances in fabricating periodic structures with high refractive index contrast, all state of the art structures are tainted by unintentional disorder. Two- and three-dimensional photonic crystals alike suffer from fluctuations in position and size of the building blocks of $2 \%-5 \%$, both for lithographically prepared [5] and self-organized structures [6]. Disorder adversely affects the interference-induced photonic crystal properties, and causes reduced Bragg reflectivity [7], extinction of transmitted light [8], and unexpected lossiness of incorporated waveguides [1]. Ultimately disorder can destroy the photonic band gap [9].

While much research has focused on fabrication and optical characterization of stop gaps, little effort has been devoted to understand the cause or effect of random multiple scattering, apart from a few studies on weakly photonic opals $[8,10,11]$. However, all light injected in 2D and 3D photonic crystals becomes diffuse after a mean free path. Hence, it is of general interest to study where diffuse photons go. Recently, exciting results have
PACS numbers: 42.70.Qs, 42.25.Dd, 42.25.Fx, 81.05.Zx

been obtained with strongly photonic inverse opals $[3,6,7,10,12]$. The mean free path in such crystals is limited to $\ell \sim 15 \mu \mathrm{m}$, owing to polydispersity, roughness, and site displacements as small as $\lesssim 1 \%$ of the cubic lattice parameter $a[6,10]$. The transport regime set by $\ell$, which is much larger than the lattice parameter, is comparable to electron transport in a conductor, as it is governed by elastic scattering due to weak disorder [13]. In this Letter we report strongly frequency-dependent, non-Lambertian angular distributions of diffuse photons transmitted by strongly photonic inverse opals in the frequency range of lowest order Bragg diffraction (L-gap). We present a model that quantitatively describes the data and for the first time unifies both disorder and photonic crystal properties. Angle-dependent internal reflection due to gaps in the dispersion plays a key role in the model. We discuss a general analysis of internal reflection based on photonic dispersion surfaces that is confirmed by our experimental observations. Our theory predicts that photons exit photonic band gap crystals in well defined diffuse beams for frequencies near band gap edges.

We have studied fcc inverse opals of titania [6], with lattice parameters $a=800,900$, and $930 \mathrm{~nm}$. These crystals consist of excellently ordered domains up to $50 \mu \mathrm{m}$ in size [Fig. 1(a)]. Front and end faces of these millimeter sized samples, with thicknesses $L \sim 200 \mu \mathrm{m}$ large compared to $\ell$, are parallel to 111 crystal planes. We illuminated the inverse opals with light over a wide frequency range $5500 \mathrm{~cm}^{-1}<\omega<14000 \mathrm{~cm}^{-1}$ from a tungsten-halogen source, which was first passed through a Fourier-transform spectrometer (Biorad FTS 6000) operated at $32 \mathrm{~cm}^{-1}$ resolution; see Fig. 1(b). The spot of $\sim 0.4 \mathrm{~mm}$ radius on the front side of the sample expanded to a diffuse "glow" distributed over all exit angles. A photodiode on a rotation stage was used to detect the diffusely transmitted photons for exit angles between $-90^{\circ}<\alpha<90^{\circ}$ relative to the surface normal 


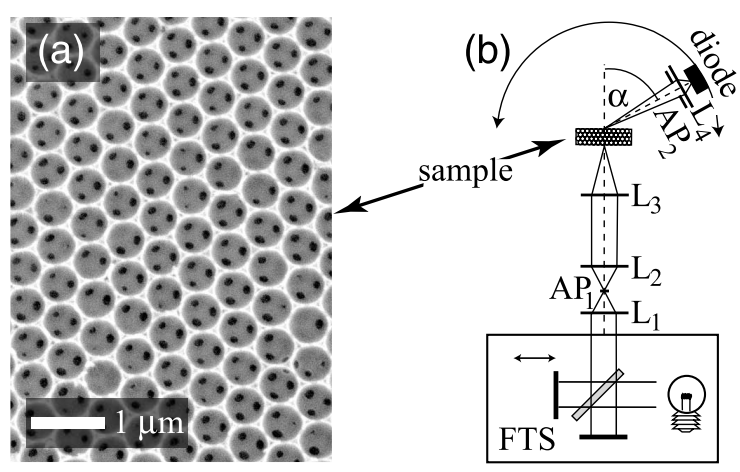

FIG. 1. (a) Scanning electron micrograph of a 111 plane of a titania inverse opal (lattice parameter $a=930 \mathrm{~nm}$ ). (b) Light from a tungsten-halogen source is passed through a Fouriertransform spectrometer (FTS), focused onto a pinhole $\mathrm{AP}_{1}$, which is imaged onto the sample. Diffuse transmission is recorded by a rotating photodiode.

with a detector acceptance angle of $5^{\circ}$. It suffices to scan $\alpha$ as the polycrystalline nature of the samples causes averaging of the threefold symmetry around the sample normal. We determined the angular probability distribution $P_{\omega}(\mu) d \mu$ for photons of frequency $\omega$ to exit the sample between angles $\alpha=\cos ^{-1} \mu$ and $\cos ^{-1}(\mu+d \mu)$ by normalizing the angle-resolved data to the total $(2 \pi \mathrm{sr}$ integrated) transmitted diffuse intensity spectrum [14].

In Fig. 2(a) we present the experimentally determined distribution $P_{\omega}(\mu)$ as a function of frequency $\omega$ and exit angle $\alpha=\cos ^{-1} \mu$ of an inverse opal with lattice spacing $a=930 \mathrm{~nm}$. For frequencies above the low frequency edge of the L-gap $\left(\omega \geqslant 7500 \mathrm{~cm}^{-1}\right)$ unusual frequencydependent angular distributions occur: a region of strongly reduced photon escape probability appears, characterized by a $70 \%$ reduction centered at a frequency of $8000 \mathrm{~cm}^{-1}$ for small angles, moving to higher frequency with increasing angle, as evident in Fig. 2(a). The large difference between these angular distributions and the well-known Lambertian distribution appropriate for ran- dom media [14] is illustrated for two key frequencies in Fig. 3(a) and 3(c). In contrast, for frequencies $\omega \lesssim$ $7000 \mathrm{~cm}^{-1}$ well below the L-gap, we find no deviation of the distribution of photons from Lambertian behavior. The center frequency and frequency width (full width at half minimum) of the gap in $P_{\omega}(\mu)$ at small angle agree well with the L-gap determined from a reflectivity experiment and the calculated band structure $[15,16]$, also shown in Fig. 2(a). Further proof of the photonic origin of the redistribution of diffuse light is the blueshift with decreasing lattice parameter. The much larger bandwidth of the gap in the angular distribution for angles $\alpha \geq 45^{\circ}$ coincides with a double-peak reflection coefficient due to coupled simultaneous diffraction by 111 and 200 planes [17], consistent with the bands in Fig. 2(a). We conclude that internal diffraction causes a strong reduction of the escape probability for diffuse photons for frequencies and angles matching a stop gap.

A remarkable feature in Fig. 2(a) is the enhanced escape probability for angles outside a stop gap, at frequencies for which a stop gap occurs at some other angle. Specifically, enhancement relative to the Lambertian distribution $P_{L}$ occurs in the frequency range $7500<\omega<$ $8500 \mathrm{~cm}^{-1}$ for angles exceeding $35^{\circ}$, and also in the frequency range above the L-gap, i.e., $9000<\omega<$ $12000 \mathrm{~cm}^{-1}$, for angles less than $30^{\circ}$. It appears that if many exit angles are forbidden due to gaps in the dispersion, the probability to escape in the remaining directions is enlarged. The magnitude and range of the escape probability enhancement cannot be understood from the photonic dispersion relation alone, which describes only the bands of internal diffraction.

To describe our data, we combine diffusion theory for internally reflecting random media [14] with a model for the angle- and frequency-dependent internal reflection coefficient $R(\mu, \omega)$ of the photonic crystals. Motivated by the good description for external reflectivity experiments [17], we take the center frequencies of the two
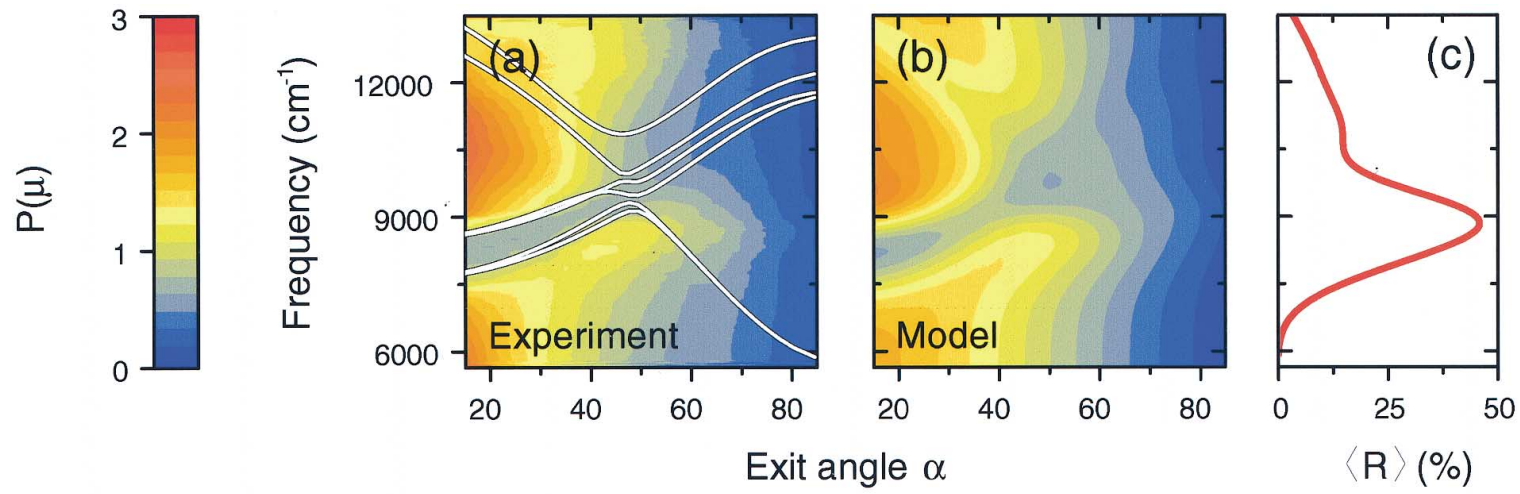

FIG. 2 (color). (a) Contour plot of the angular distribution of transmitted photons versus frequency, measured on an inverse opal with lattice spacing $a=930 \mathrm{~nm}$. White curves show the lowest six dispersion bands along the LU line, compensated for refraction. (b) Contour plot of the calculated angular distribution, using diffusion theory with internal reflections determined by the band structure. (c) The angle-averaged reflectivity $\langle R\rangle$ according to the diffusion model. $\langle R\rangle$ determines the diffuse transmission enhancement outside a stop gap direction. 
relevant Gaussian reflectivity peaks from the band structure along the LU line from $\mathrm{L}$ to $\mathrm{U}$ point in the Brillouin zone [Fig. 2(a)]. We have adjusted the magnitudes of the peak reflectivities to obtain an optimal fit [Fig. 2(b)] to the data, agreeing to within $10 \%$ over the full angle and frequency range. The good agreement between data and the complete model is illustrated for two key frequencies in Figs. 3(a) and 3(c). For angles $\alpha=\cos ^{-1} \mu$ outside a stop gap, the enhancement can simply be expressed as

$$
\Delta P_{\omega}(\mu)=P_{\omega}(\mu)-P_{L}(\mu)=\mu \mathcal{E}_{\omega}=\mu \frac{2\langle R\rangle(\omega)}{1-\langle R\rangle(\omega)},
$$

where $P_{L}(\mu)=\mu\left(1+\frac{3}{2} \mu\right)$. The enhancements of the diffuse transmission depend on one single parameter, which is a frequency-dependent, angle-averaged reflection coefficient $\langle R\rangle(\omega)$ [14]. More generally, the escape probability
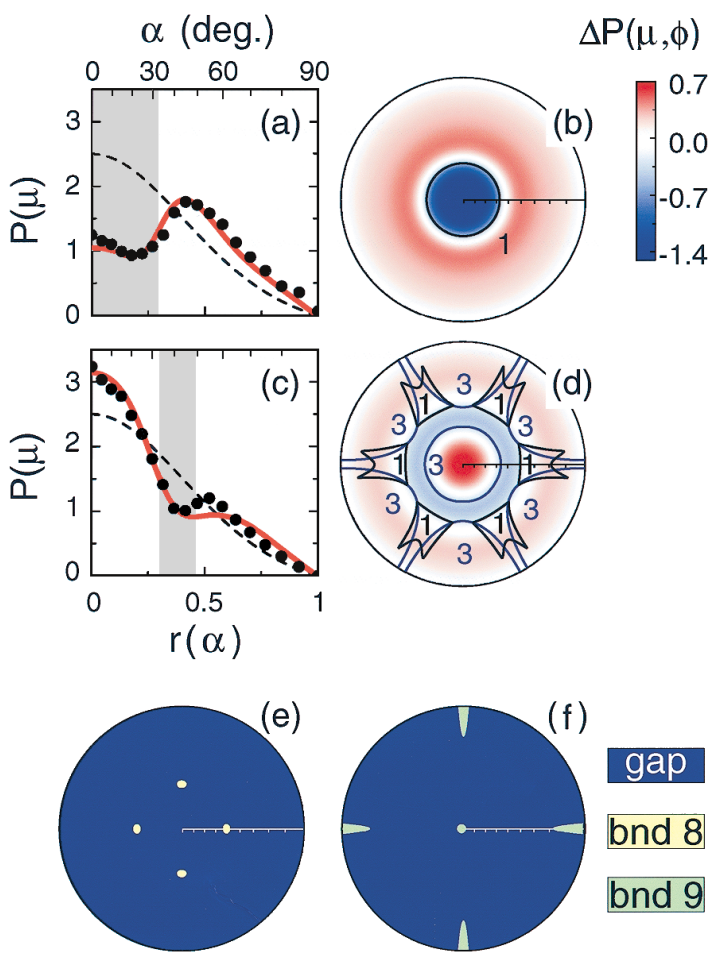

FIG. 3 (color). Photon exit distribution $P(\mu)$ for frequencies $\omega=8200 \mathrm{~cm}^{-1}$ (a) and $9300 \mathrm{~cm}^{-1}$ (c) measured for a 111 cleaved inverse opal with $a=930 \mathrm{~nm}$. Both the data (dots) and the adapted diffusion model (red curves) strongly differ from the Lambertian distribution $P_{L}$ (dashed line). Grey areas indicate forbidden exit angles according to the dispersion surface analysis. (b),(d) Stereographic plots of the photon excess distribution $\Delta P(\mu)=P(\mu)-P_{L}(\mu)$, corresponding to the models in (a) and (c), respectively. Solid curves in (b) and (d) indicate boundaries of regions in exit angle space, numbered with the band index, for which coupling to a photonic single crystal is possible. Bands 2 and 4 are similar to bands 1 and 3, respectively. For a 100-cleaved inverse opal with $\epsilon=$ 11.9 , the only allowed exit angles are indicated in yellow $[\omega a / 2 \pi c=0.776$ below the full band gap (e) $]$ or green $[\omega a / 2 \pi c=0.816$ above the band gap (f)].

$$
P_{\omega}(\mu)=\mu\left[\left(\mathcal{E}_{\omega}+1\right)+\frac{3}{2} \mu\right][1-R(\mu, \omega)]
$$

is reduced in direct correspondence to the angle-resolved internal reflection coefficient $R(\mu, \omega)$ set by the gaps in the dispersion relation. At a given frequency $\omega$, the diffuse transmission is strongly directional if most angles are contained in a stop gap. For all angles in a stop gap, the diffuse transmission is suppressed by internal reflection [large $R(\mu, \omega)$ ], while for the remaining angles the angular distribution function is strongly enhanced due to the high average reflection coefficient $\langle R\rangle$ [via the factor $\left.\mathcal{E}_{\omega}\right]$. For the titania inverse opals this results in a pronounced maximum $\langle R\rangle \sim 50 \%$ at the blue edge of the L-gap, in contrast to the frequency-independent $\langle R\rangle$ in random media [cf. Fig. 2(c)].

The scope of the diffusion calculation is limited by our empirical approximation for the ranges of internal diffraction, i.e., for the ranges of $\mu$ and $\omega$ for which $R(\mu, \omega) \sim 1$. To overcome these limitations, we proceed with a general analysis of the exit angles for which coupling to any Bloch mode is impossible at a given frequency. Such an analysis is composed of three steps [18]: (1) determining the photon dispersion surfaces in the photonic crystal, i.e., the constant frequency surfaces in $\mathbf{k}$ space, (2) applying parallel momentum conservation $\mathbf{k}_{\| \text {,crystal }}=\mathbf{k}_{\| \text {,out }}$. Because of the discrete translational symmetry along the crystal surface [19] one should either consider dispersion surfaces in the repeated zone scheme or equivalently allow modes with $\left(\mathbf{k}_{\text {crystal }}+\mathbf{G}\right)_{\|}=\mathbf{k}_{\| \text {,out }}$ for any reciprocal lattice vector G. Finally, (3) Bloch modes for which the group velocity does not point towards the crystal interface are discarded. We have determined dispersion surfaces and group velocities by interpolating from eigenfrequencies calculated on a dense $\mathbf{k}$ grid within the volume and on the facets of the Brillouin zone [15,20].

The excellent agreement of the general refraction construction with the experiment is demonstrated in Figs. 3(b) and 3(d), for the two frequencies corresponding to the data in 3(a) and 3(c). In these stereographic plots [21] only the boundaries of the theoretically forbidden regions in exit-angle space are indicated. For a frequency in the L-gap $\omega=8200 \mathrm{~cm}^{-1}$ [Fig. 3(b)] coupling is allowed only to band 1 and the nearly polarization degenerate band 2 , and is limited to $\alpha>33^{\circ}$, independent of the azimuthal angle $\phi$. This is in perfect agreement with the measured photon excess $\Delta P_{\omega}(\mu)$ [color scale in Fig. 3(b)], which is negative for $0<\alpha<35^{\circ}$ and enhanced for $\alpha>35^{\circ}$. For a higher frequency above the L-gap [ $\omega=9300 \mathrm{~cm}^{-1}$, Fig. 3(d)] coupling to bands 1 and 2 is only allowed in six small lobes in exit-angle space. For angles $\alpha<34.5^{\circ}$ coupling occurs to bands 3 and 4. For larger angles $\alpha>51^{\circ}$, six parabolas delimit angular ranges in which coupling from bands 3 and 4 to air is allowed. The only angles to which diffuse light inside the samples cannot couple are contained in a ring concentric with the origin, and six patches with 
$80^{\circ}<\alpha<90^{\circ}$ and azimuthal width $\lesssim 6^{\circ}$, too small to affect the experimental azimuthal average. The boundaries of the ring are sixfold symmetric, but not quite circular. The agreement of the central forbidden ring with the experimental data for $\Delta P_{\omega}(\mu)$ is again gratifying [Fig. 3(d)].

Motivated by the close agreement of the dispersion surface analysis with the experimentally determined diffuse transmission of strongly photonic crystals, we consider the interesting possibilities offered at frequencies near photonic band gap edges. For frequencies near the edge of any photonic band gap that closes away from the center of the Brillouin zone, all modes have wave vectors in pockets away from the $\mathbf{k}$-space origin. In such cases diffuse light may exit the photonic crystal only along isolated directions. As the shape of dispersion surfaces for frequencies near a band gap is nearly ellipsoidal, an effective mass approximation is well suited to predict the directionality of diffuse transmission. Recently, inverse opals of silicon have been prepared by several groups $[22,23]$. We therefore consider the diffuse transmission of such an inverse opal with a full photonic band gap, assuming a $\epsilon=11.9$ backbone containing fcc closepacked air spheres. In Fig. 3(e) the solution of the refraction problem for a 100 cleaved crystal $[22,23]$ is shown for a frequency $\hat{\omega}=\omega a / 2 \pi c=0.776$ just below the band gap. In the frequency range from $\hat{\omega}=0.748$ up to the band gap edge $\hat{\omega}=0.778$ the only allowed modes are in band 8, and the dispersion surfaces are ellipsoids around the $W$ point. Diffuse light inside the crystal can couple only into a symmetric quadruplet of beams, with $\alpha \sim 40^{\circ}$. For frequencies above the band gap (limited by band 9 at the $X$ point) emission is directed into a central beam along the surface normal, with a width proportional to the square root of the detuning from the gap edge. The four beams at grazing exit angle [Fig. 3(f)] do not appear in a small frequency range just above the gap edge due to total internal reflection. At these frequencies all light inside the crystal, be it multiply scattered from an external source or emission from inside the crystal, may leave the crystal in only one single narrow beam of diffuse light. This phenomenon is robust against polycrystalline averaging. Directional diffuse beams will occur whenever the gap closes away from the k-space origin, e.g., for diamond, hcp, fcc, and bcc structures.

The analysis of the angle-resolved diffuse intensity distribution to study photonic dispersion appears analogous to high-resolution angle-resolved photoemission spectroscopy. This powerful technique is instrumental in the study of surface and projected bulk electronic band structures and Fermi surfaces in, e.g., metals or high $T_{c}$ superconductors [24]. Within this analogy, the optical probe frequency can be identified with the Fermi energy of the electron. Moreover, the optical experiment should be considered a zero-temperature analogon of the electronic case. Experimentally, the probe depth in the photonic crystals, $\ell \leqq 10$ unit cells, appears less restricted to the surface than in photoemission.

We thank Peter Lodahl and Ad Lagendijk for stimulating discussions. This work is part of the research program of the "Stichting voor Fundamenteel Onderzoek der Materie (FOM)," which is financially supported by the "Nederlandse Organisatie voor Wetenschappelijk Onderzoek (NWO)."

*Electronic addresses: femius.koenderink@phys.chem. ethz.ch; www.photonicbandgaps.com

[1] Photonic Crystals and Light Localization in the 21st Century, edited by C. M. Soukoulis (Kluwer, Dordrecht, 2001).

[2] E. Yablonovitch, Phys. Rev. Lett. 58, 2059 (1987).

[3] A. F. Koenderink et al., Phys. Rev. Lett. 88, 143903 (2002).

[4] S. John, Phys. Rev. Lett. 58, 2486 (1987).

[5] T. Baba and N. Fukaya, in Ref. [1], pp. 105-116; M. Notomi et al., Phys. Rev. Lett. 87, 253902 (2001); S. Ogawa, K. Tomoda, and S. Noda, J. Appl. Phys. 91, 513 (2002).

[6] J. E. G. J. Wijnhoven and W. L. Vos, Science 281, 802 (1998); J. E. G. J. Wijnhoven, L. Bechger, and W. L. Vos, Chem. Mater. 13, 4486 (2001).

[7] J. F. Galisteo Lòpez and W. L. Vos, Phys. Rev. E 66, 036616 (2002).

[8] Y. A. Vlasov, M. A. Kaliteevski, and V.V. Nikolaev, Phys. Rev. B 60, 1555 (1999); V. N. Astratov et al., ibid. 66, 165215 (2002).

[9] M. M. Sigalas et al., Phys. Rev. B 59, 12767 (1999); Z. Y. Li and Z. Q. Zhang, ibid. 62, 1516 (2000).

[10] A. F. Koenderink et al., Phys. Lett. A 268, 104 (2000).

[11] J. Huang et al., Phys. Rev. Lett. 86, 4815 (2001).

[12] M. S. Thijssen et al., Phys. Rev. Lett. 83, 2730 (1999).

[13] P. A. Lee and T. V. Ramakrishan, Rev. Mod. Phys. 57, 287 (1985); M. C.W. van Rossum and Th. M. Nieuwenhuizen, ibid. 71, 313 (1999).

[14] J. X. Zhu, D. J. Pine, and D. A. Weitz, Phys. Rev. A 44, 3948 (1991); D. J. Durian, Phys. Rev. E 50, 857 (1994).

[15] The H-field method with 725 plane waves was used. See K. Busch and S. John, Phys. Rev. E 58, 3896 (1998).

[16] $\mathrm{TiO}_{2}$ crystals were modeled as fcc close-packed air spheres surrounded by high index $(\epsilon=6.5)$ shells (inner radius $r=a / \sqrt{8}$, outer radius $1.09 r$ ) connected by cylindrical windows of radius $0.4 r$. See Ref. [3].

[17] H. M. van Driel and W. L. Vos, Phys. Rev. B 62, 9872 (2000).

[18] M. Notomi, Phys. Rev. B 62, 10696 (2000).

[19] This is valid only for cleavages along a crystal plane.

[20] We used 2992 equidistant $k$ points within the irreducible part of the Brillouin zone (BZ), 1816 points on the 111 facet and 1255 on the 200 facet of the BZ.

[21] The stereographic radial position is $r=\sin \alpha /(\cos \alpha+1)$.

[22] E. Palacios-Lidón et al., Appl. Phys. Lett. 81, 4925 (2003).

[23] Y. A. Vlasov, X. Z. Bo, J.C. Sturm, and D. J. Norris, Nature (London) 414, 289 (2001).

[24] S. Hüfner, Photoelectron Spectroscopy: Principles and Applications (Springer-Verlag, Berlin, 1995). 